\title{
Magnetic Micropillar Sensors for Force Sensing
}

\author{
A. Alfadhel*, J. Kosel \\ Computer, Electrical and Mathematical Sciences and Engineering Division (CEMSE) \\ King Abdullah University of Science and Technology (KAUST) \\ Thuwal, Saudi Arabia \\ *Email: ahmed.fadhel@kaust.edu.sa
}

\begin{abstract}
A force sensor system consisting of bioinspired, magnetic and highly elastic micropillars integrated on a magnetic field sensing element is reported. The micropillars are made of a nanocomposite consisting of magnetic nanowires incorporated into polydimethylsiloxane. The permanent magnetic behavior of the nanowires allows remote operation without an additional magnetic field to magnetize the nanowires, which simplifies miniaturization and system integration. We demonstrate the potential of this concept by realizing a tactile sensing element. The developed sensor element operates at power consumption of $75 \mu \mathrm{W}$ and has a detection range between $0-120 \mathrm{kPa}$ and a resolution of $2.7 \mathrm{kPa}$, which can easily be tuned in a wide range.
\end{abstract}

Keywords- Force Sensor, Nanocomposite, Nanowires, Magnetic, Polymer, Cilia, Microfabrication, Tactile sensor

\section{INTRODUCTION}

The current progress in fields like consumer electronics, robotics or biomedical engineering requires the development of sensing technologies that combine miniaturization with a high sensitivity and low power consumption. Force sensors with different sensing mechanisms have been introduced such as resistive, piezoelectric and capacitive sensing [1-6]. Many technical challenges still remain in current tactile and force sensing research, including low sensitivity, high power consumption, in addition to the interference with environmental noise.

Advances in material integration and nanofabrication technologies have enabled new directions of research and development for microsensor systems. Examples are nanocomposite materials, which combine the advantages of polymers like flexibility, elasticity, chemical resistance or biocompatibility with the unique properties of nanoparticles. Magnetic nanocomposites have been introduced before using superparamagnetic particles [7]. However, these particles require a high magnetic field for magnetization, which increases the power consumption and limits the possibility of integration and miniaturization. On the other hand, iron nanowires (NWs) have a high magnetization in the absence of a magnetic field, due to the strong shape anisotropy, and they can be easily fabricated with a cost effective process [8]. Incorporating these NWs in a polymer results in a permanent magnetic composite with distinct features and reinforced mechanical properties [9].

We report the fabrication and characterization of a force sensor that consists of magnetic micropillars integrated on a magnetic field sensing element for a wide range of sensing applications including tactile sensing for robotics or smart gloves, smart prosthesis, surgical tools, or flow sensing. The micropillars are fabricated from a nanocomposite that is made of magnetic NWs incorporated into polydimethylsiloxane
(PDMS). The developed nanocomposite utilizes the permanent magnetic behavior of the NWs, is highly elastic, can easily be patterned and has a good corrosion resistance.

\section{METHODS}

\section{A. Sensor Concept}

The operating principle of the sensor is based on detecting the change of the magnetic field, created by the permanent magnetic NWs, of the magnetic micropillars, when deflected by an external force (e.g. fluid flow or hand touch) (Fig. 1). A multi-layer giant magneto-impedance (GMI) sensor, which offers a good compromise between sensitivity and fabrication complexity [10-13], is utilized to measure the change of the magnetic field. At small bias fields and at high operating frequencies, the impedance of GMI sensors is sensitive to external magnetic fields, due to the strong dependence of the skin effect on the magnetic permeability. A distinct advantage of the nanocomposite pillars is the permanent magnetic behavior of the iron NWs, eliminating the need for an external magnetic field to magnetize the pillars or bias the GMI sensor. At resting position, the stray field of the pillars affects the GMI sensor with an average magnetic field value that biases the sensor and changes its initial impedance. In the presence of a force, the pillars bend in the force direction. This bending results in a change of the stray field of the pillars with an average magnetic field value affecting the GMI sensor, and hence changing its impedance.

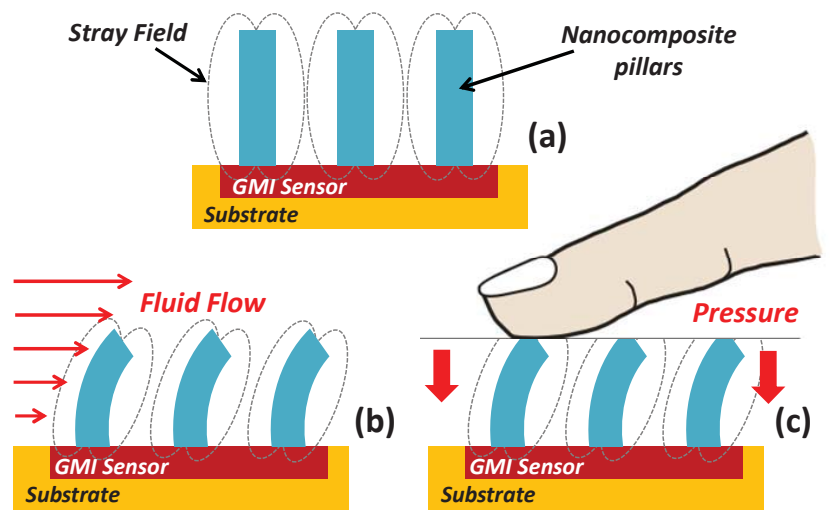

Fig. 1. Illustration of the operating principle of a magnetic micropillar force sensor. (a) The sensor element is made of permanent magnetic nanocomposite pillars integrated on a giant magnetoimpedance (GMI) sensor. The pillars get deflected by external forces such as fluid flow (b) or hand touch (c) changing the stray field at the GMI sensor, and hence changing its impedance.

The deflection $(\delta)$ of the pillars due to a shear or horizontal force $F$ is

$$
\delta=\frac{64}{3} F \frac{l^{3}}{\pi E D^{4}}
$$


where $l, E$, and $D$ are the length, the Young's modulus and the diameter of the pillars, respectively. Hence, the detection range and sensitivity can be easily controlled by modifying the mechanical properties of the nanocomposite pillars, i.e. the length, width or Young's modulus.

As an example of a potential application, a flow sensor has been reported previously using the same sensing concept [14]. $500 \mu \mathrm{m}$ long and $100 \mu \mathrm{m}$ in diameter pillars were fabricated on an $8 \mathrm{~mm}$ long and $400 \mu \mathrm{m}$ wide GMI sensor. The flow sensor is tested inside a fluidic channel. The sensor shows outstanding performance with very low power consumption and high sensitivity, in addition to the proven advantages such as the easy fabrication and good corrosion resistance.

\section{B. Fabrication}

The NWs-PDMS nanocomposite serves as the building block of the reported sensing concept. Iron NWs are fabricated by the electrodeposition into nanoporous aluminum oxide membrane, prepared by a two-step anodization process using oxalic acid on an aluminum substrate. Iron is chosen for its high magnetization at remanence and high coercivity, acting as nanoscale permanent magnets and making the pillars hard to demagnetize. Additionally, iron NWs are biocompatible and can be efficiently fabricated with a high control of the properties. The PDMS nanocomposite contains iron NWs of 6 $\mu \mathrm{m}$ in length and $35 \mathrm{~nm}$ in diameter. The nanocomposite is prepared by mixing iron NWs dispersed in Sodium dodecyl sulfate (SDS) surfactant in PDMS (Sylgard 184 Silicone Elastomer, Dow Corning Corporation) that is used as the polymeric matrix. The nanocomposite is typically cured at $90^{\circ} \mathrm{C}$ for 1 hour. Different NWs concentration can be used and the alignment direction of the NWs can be easily controlled by applying a homogenous magnetic field during the curing process and along the desired direction (Fig. 2).
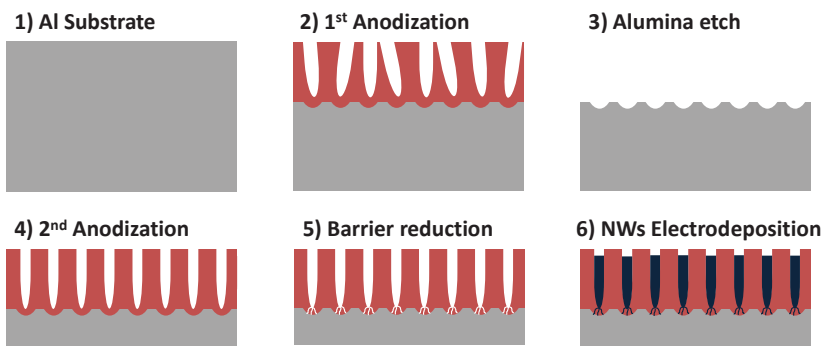

6) NWs Electrodeposition

7) NWs Release

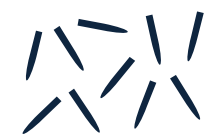

8) NWs mixing with polymer
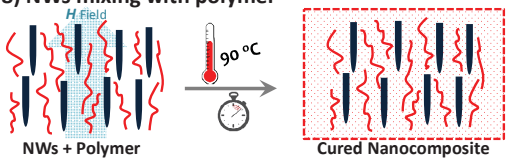

Fig. 2. Illustration of the electrochemical fabrication process of iron NWs and the process of fabricating the nanocomposite. Aluminum substrate is used in the process (1) and two anodization steps are performed using oxalic acid to obtain uniform nanopores (2-4) of $\sim 30 \mathrm{~nm}$ in diameter, followed by a barrier reduction step for establishing an electrical contact with the bottom electrode (5). Iron is electrodeposited into the nanopores (6) and then the NWs are released by etching the Alumina membrane and dispersed in ethanol (7). The nanocomposite is fabricated by mixing the NWs with the polymer and then curing the composite. The NWs can be aligned in a certain direction by applying an external homogenous magnetic field during the curing process (8).

A three-layer GMI magnetic field sensor that has a meander structure of $2 \times 2 \mathrm{~mm}^{2}$ and has $2 \mathrm{~mm}$ long and $200 \mu \mathrm{m}$ wide conductors with $200 \mu \mathrm{m}$ spacing is fabricated to detect

the change of the stray magnetic field. The GMI sensor is composed of $200 \mathrm{~nm}$ thick $\mathrm{Cu}$ layer sandwiched by two 100 $\mathrm{nm}$ thick $\mathrm{Ni}_{80} \mathrm{Fe}_{20}$ layers. The GMI sensor structure is patterned onto a substrate using standard lithography and the materials are deposited by e-beam evaporation with a constant magnetic field of 100 Oe applied in the transverse direction to induce a uniaxial magnetic anisotropy, creating a magnetically sensitive axis in the longitudinal direction, and then followed by a lift-off process.

The nanocomposite has 20\% NWs:PDMS volume ratio. The NW ratio in the nanocomposite is chosen to provide the biasing field for the GMI sensor and, at the same time, to not adversely affect the polymerization process of the PDMS or the elasticity of the pillars. Nanocomposite pillars are fabricated using a master mold technique. A $1 \mathrm{~mm}$ thick poly(methylmethcrylate) (PMMA) master mold into which an array of holes with $200 \mu \mathrm{m}$ in diameter is patterned with a $\mathrm{CO}_{2}$ laser cutter (Universal PLS6.75). The composite is casted onto the surface of the GMI sensor and the master mold is mounted on top with aligning the holes along the GMI sensor using a microscope. This structure is then placed in a desiccator for 30 minutes to remove any trapped air bubbles and assist in filling the pores. Next, the composite is cured at 90 degrees Celsius for one hour with external magnetic field along the length of the pillar, forming the pillars with aligned NWs on top of the GMI sensor. After releasing the cured pillars, they are fully magnetized by applying a magnetic field of $10 \mathrm{kOe}$. The pillars are arranged on the GMI sensor such that they can almost fully deflect and are placed along conductors with same current direction. The fabrication process is illustrated in Fig. 3. (a)

Photoresist exposure and development
Photoresist
Substrate
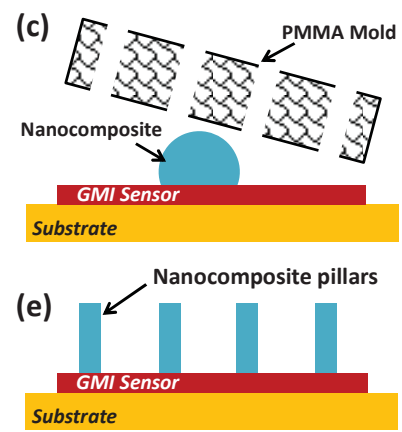

(b)

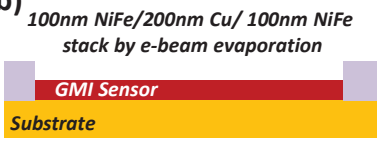

(d)

PMMA mold placed on the nanocomposite and cured at $90^{\circ} \mathrm{C}$ for 1 hour

NWs alignment performed while curing

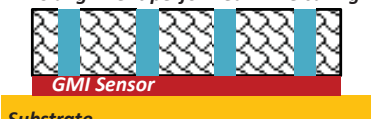

Substrate

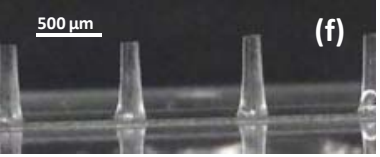

Fig. 3. Fabrication process of the magnetic micropillar force sensor. (a,b) A giant magnetoimpedance (GMI) sensor is fabricated by depositing $100 \mathrm{~nm}$ permalloy/ $200 \mathrm{~nm} \mathrm{Cu} / 100 \mathrm{~nm}$ permalloy by e-beam evaporation and it is patterned using a standard lithography process. (b-e) Micropillars are fabricated using a PMMA mold. (f) Micropillars with $500 \mu \mathrm{m}$ in length and $100 \mu \mathrm{m}$ in diameter.

\section{Characterization}

In order to test the effect of the NWs' alignment on the magnetic properties of the nanocomposite, the magnetization curves along the length of the pillars and along their perpendicular direction are obtained using a vibrating sample magnetometer. As shown in Fig. 4, the pillars with vertically aligned NWs have a magnetic anisotropy with a remanence to saturation magnetization of $95 \%$ along the vertical direction 
and $37 \%$ along the horizontal direction with a coercivity of $1513 \mathrm{kOe}$. These results indicate the permanent magnetic like behavior of the nanocomposite. Transmission electron microscopy imaging is conducted to study the distribution of NWs in pillars' cross section as shown in the inset $f$ Fig. 4 NWs X-ray diffraction analysis reveals the NWs are polycrystalline iron with a thin shell of magnetite around the NWs (Fig. 5).

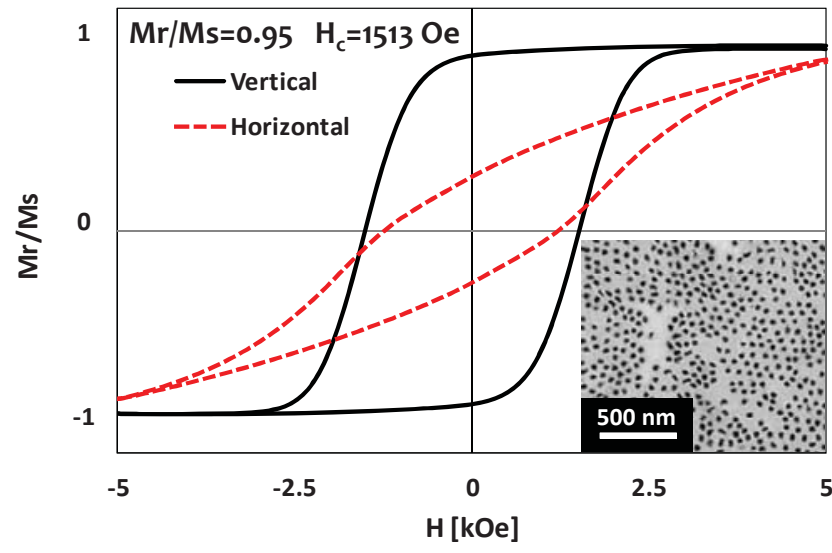

Fig. 4. Magnetization curves of magnetic micropillars obtained with a vibrating sample magnetometer in the vertical and horizontal direction of pillars with vertically aligned NWs. Inset: Transmission electron microscopy image of the pillars' coross section showing the distribution of the NWs in the nanocomposite.

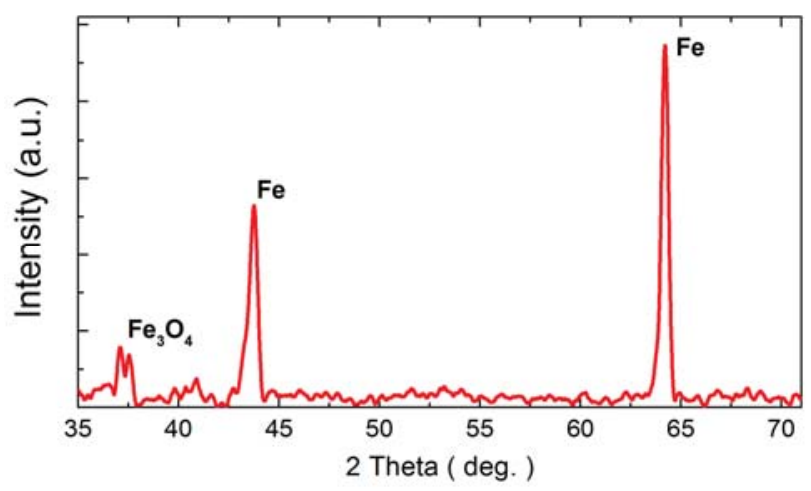

Fig. 5. X-ray diffraction results for a nanocomposite with iron nanowires.

In order to characterize the GMI sensor, a Helmholtz coil is used to apply a magnetic field from 0 Oe to $60 \mathrm{Oe}$, while the impedance is obtained with an impedance analyzer (Agilent E4991A) at a current of $0.1 \mathrm{~mA}$ in amplitude at a frequency of $200 \mathrm{MHz}$. The fields are applied in the longitudinal and transverse direction (Fig. 6). The GMI sensor response shows a peak at a field of 10 Oe corresponding to the anisotropy field of the $\mathrm{Ni}_{80} \mathrm{Fe}_{20}$ thin film. As can be seen, the GMI sensor is magnetically sensitive in the longitudinal direction with maximum sensitivity of $0.2 \Omega / \mathrm{Oe}$, while no significant impedance change is observed in the transverse direction. This anisotropic magnetic property of the GMI sensor provides a good selectivity in terms of the direction of the magnetic signal that is being measured.

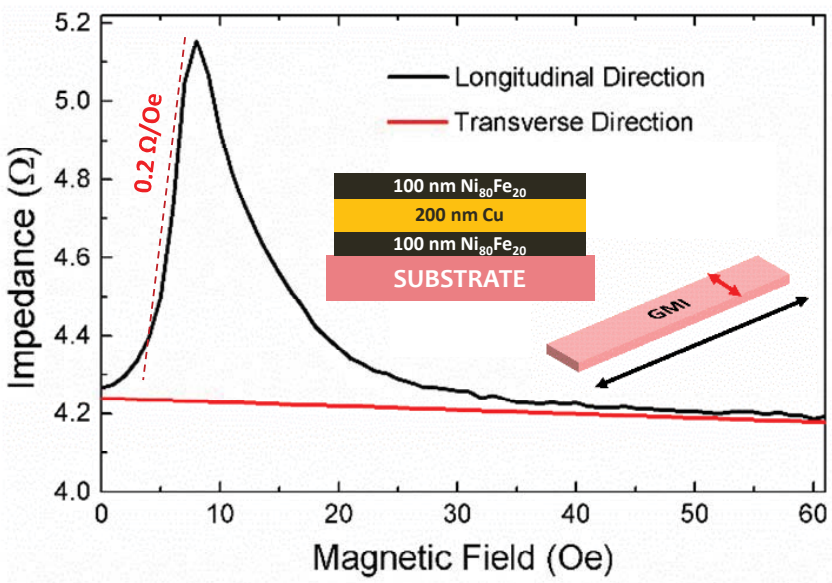

Fig. 6. Impedance response of the giant magnetoimpedance (GMI) sensor with an external magnetic field applied in the sensitive (longitudinal) and nonsensitive (transverse) directions at $200 \mathrm{MHz}$. The GMI sensor's sensitivity is determined by a linear fit.

\section{RESULTS}

The force sensor is tested by applying vertical loads using PMMA weights, where the pressure is calculated by considering the cross section of the pillars. The signal is obtained through a balanced Wheatstone bridge configuration made of four identical sensors, where the load is applied to a single sensor only acting as the active element as shown in Fig. 7. An RF waveform generator (Agilent E8257D) is used to power the sensor with a sinusoidal signal $\left(V_{s}\right)$ of $50 \mathrm{mV}$ amplitude and a frequency of $200 \mathrm{MHz}$. The bridge signal $\left(\mathrm{V}_{\mathrm{g}}\right)$ at different loads is measured using an RF lock-In amplifier (Stanford SR844) at $200 \mathrm{MHz}$ center frequency, 30 ms time constant, and $18 \mathrm{~dB} /$ Octave filter slope. All data are stored using a LabVIEW program. $V_{g}$ can be estimated using:

$$
V_{g}=\left(\frac{Z_{\text {sensor }}}{Z_{\text {ref }}+Z_{\text {sensor }}}-\frac{Z_{r e f}}{2 \times Z_{r e f}}\right) V_{s},
$$

where $Z_{\text {sensor }}$ is the impedance of the force sensor, and $Z_{\text {ref }}$ is the impedance of the other balancing sensors in the Wheatstone bridge. Small signal offset $\left(V_{\text {off }}\right)$ in the bridge of $0.2 \mathrm{mV}$ amplitude is obtained at no load applied which could be due to the signal interference at high frequency. To represent the signal of the sensor only, the signal is considered such as:

$$
\Delta V=V_{g}-V_{\text {off }}
$$

As shown in Fig. 8, the force sensor element can detect a pressure range of $0-120 \mathrm{kPa}$ with a resolution of $2.7 \mathrm{kPa}$. A linear range from $0 \mathrm{kPa}$ to $32 \mathrm{kPa}$ is observed with a sensitivity of $0.55 \mu \mathrm{V} \cdot \mathrm{kPa}^{-1}$. Another linear region from 32 $\mathrm{kPa}$ to $120 \mathrm{kPa}$ is obtained with a sensitivity of $0.1 \mu \mathrm{V} \cdot \mathrm{kPa}^{-1}$. Response saturation is obtained at $23 \mu \mathrm{V}$ representing the full deflection of the pillars. Noise in the system is about $\pm 1.2 \mu \mathrm{V}$.

The average power consumption of the force sensor, when operated at $6 \mathrm{~mA}$ current amplitude in the bridge structure, is $75 \mu \mathrm{W}$. The power consumption can be further reduced by operating the sensor at current amplitudes as low as $0.1 \mathrm{~mA}$ reducing the power consumption to $20 \mathrm{nW}$ with almost no influence of the current on the measured impedance values as discussed in [14]. 


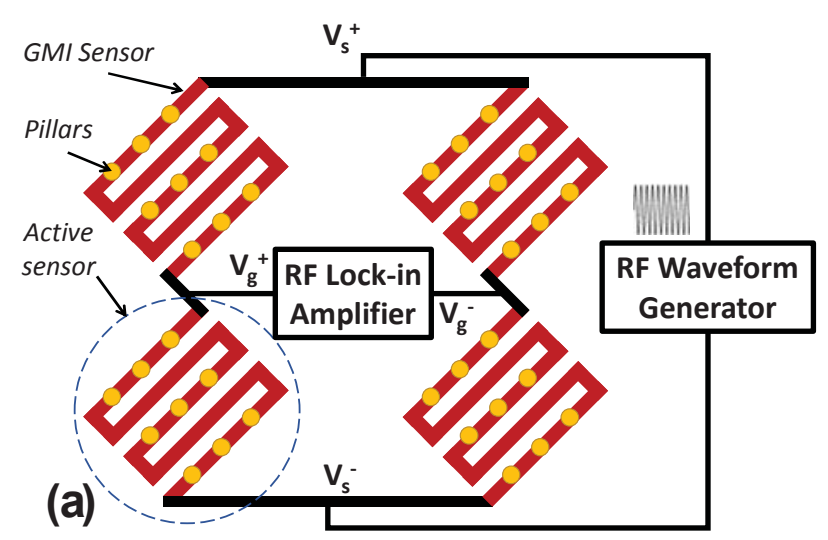

Fig. 7. Experimental setup of the force sensor using a Wheatstone bridge configuration to measure the voltage response.

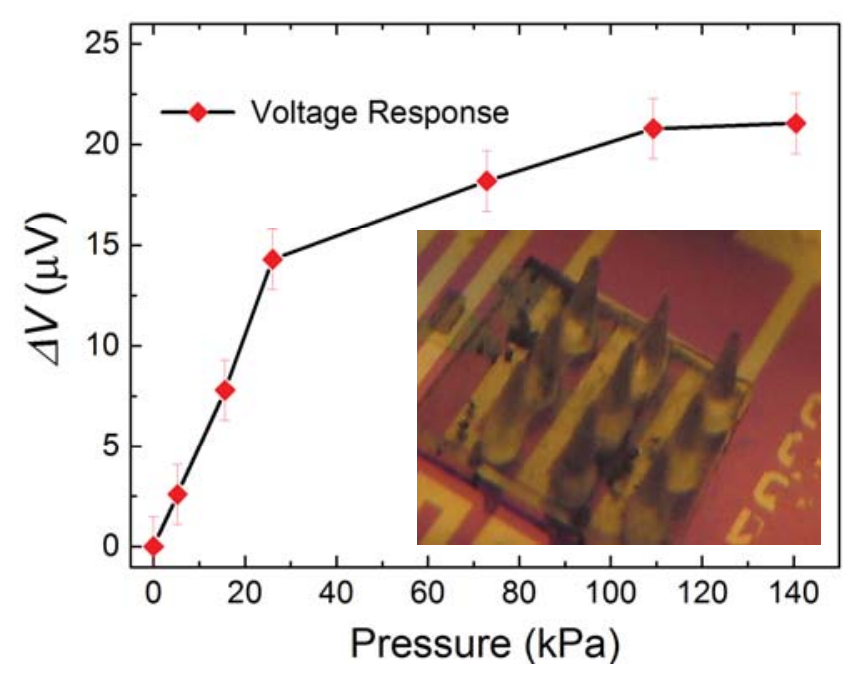

Fig. 8. Experimental voltage change of the force sensor in the Wheatstone bridge configuration at different applied pressures showing the error bars for the signal fluctuation due to the noise in the system. Inset: Image of the fabricated sensor.

\section{CONCLUSION}

A NWs-based PDMS permanent magnetic and highly elastic nanocomposite has been used to realize micropillars on top of a GMI sensor. This cilia sensor concept is used for force measurements as is typical in, e.g., flow measurements or tactile sensing. The permanent magnetic behavior of the NWs enables detecting the deflection of the pillars due to a force with the GMI sensor, and biasing the GMI sensor at its optimal operation point. This greatly simplifies miniaturization, aids integration and minimizes the power consumption. The magnetic properties of the nanocomposite can be tailored in a wide range by the choice of the materials and the dimensions of the polymer and NWs as well as by the concentration of the NWs. An additional parameter to adjust the performance with is the alignment of the NWs in the nanocomposite pillars, which can be exploited to create isotropic or anisotropic behavior. Three-layer GMI sensors are used because they offer a good compromise between sensitivity and fabrication complexity. Moreover, they can be implemented as wireless devices, which can be utilized to realize passive and remote sensors.

The results of the tactile sensor show a detection range of 0 $120 \mathrm{kPa}$ with resolution of $2.7 \mathrm{kPa}$ and a high sensitivity of $0.55 \mu \mathrm{V} \cdot \mathrm{kPa}^{-1}$ between $0-32 \mathrm{kPa}$, and sensitivity $0.1 \mu \mathrm{V} \cdot \mathrm{kPa}^{-1}$ between $32-120 \mathrm{kPa}$. The pillars buckle easily in the first region and sensitivity reduces as it gets harder to fully deflect the pillars until reaching the maximum deflection resulting in signal saturation. The developed sensor has easily modifiable sensing range and resolution. The power consumption of the sensor is $75 \mu \mathrm{W}$ and can be reduced as low as $20 \mathrm{nW}$. Nine pillars were used in the current sensor design, which were arranged in the meander GMI sensor in a manner allowing full deflection with considering the sensitivity direction and the current direction. This design provides an average signal over the $2 \mathrm{~mm}$ by $2 \mathrm{~mm}$ meander sensor and can be readily adjusted to meet other requirements.

\section{ACKNOWLEDGEMENTS}

Research reported in this publication was supported by the King Abdullah University of Science and Technology (KAUST).

\section{REFERENCES}

[1] S. Mohsin, I. Tiwana, and N. Lovell, "A review of tactile sensing technologies with applications in biomedical engineering," Sens. Actuators A, vol. 179, pp. 17, 2012.

[2] K. Takei, et al., "Nanowire active-matrix circuitry for low-voltage macroscale artificial skin," Nature Materials, vol. 9, pp. 821, 2010.

[3] C. Pang, et al., "A flexible and highly sensitive strain-gauge sensor using reversible interlocking of nanofibres," Nature Materials, vol. 11, pp. 795, 2012.

[4] B. Tee, et al., "An electrically and mechanically self-healing composite with pressure- and flexion-sensitive properties for electronic skin applications," Nature Nanotechnology, vol. 7, pp. 825, 2012.

[5] M. Segev-Bar, A. Landman, M. Nir-Shapira, G. Shuster, and H. Haick, "Tunable Touch Sensor and Combined Sensing Platform: Toward Nanoparticle-based Electronic Skin,"ACS Applied Mater. \& Inter., vol. 5, pp. 5531, 2013.

[6] H. Kim, S. Lee, and K. Yun, "Capacitive tactile sensor array for touch screen application,” Sens. and Actuators A, vol. 165, pp. 2, 2011.

[7] M. Suter, et al., "A photopatternable superparamagnetic nanocomposite: Material characterization and fabrication of microstructures," Sens. and Actuat. B, vol. 156, pp. 433-443, 2011.

[8] S. Thongmeea, H.L. Panga, J. Dinga, and J.Y. Lin "Fabrication and magnetic properties of metallic nanowires via AAO templates," $J$. of Mag. and Mag. Mat., vol. 321, pp. 2712-2716, 2009.

[9] K. Keshoju and L. Sun, "Mechanical characterization of magnetic nanowire-polydimethylsiloxane composites," J. Appl. Phys., vol. 105, pp. $023515,2009$.

[10] L. Panina, and K. Mohri, "Magneto-impedance in multilayer films," Sensors Actuators A, vol. 81, pp. 71-77, 2000.

[11] B. Li, et al., "Integration of thin film giant magneto impedance sensor and surface acoustic wave transponder," J. Appl. Phys., vol. 111, pp. 07E514, 2012.

[12] B. Li, and J. Kosel, "Three dimensional simulation of giant magnetoimpedance effect in thin film based sensors," Journal of Applied Physics, 109, pp. 07E519, 2011.

[13] B. Li, A. Morsy, and J. Kosel, "Optimization of autonomous magnetic field sensor consisting of giant magnetoimpedance sensor and surface acoustic wave transducer," IEEE Trans. Magnetics, 48, vol. 11, pp. 4324, 2012.

[14] A. Alfadhel, B. Li, A. Zaher, O. Yassine, and J. Kosel, "Magnetic nanocomposite for biomimetic flow sensing," Lab Chip, vol. 14, pp. 4362-4369, 2014. - Reproduced by permission of The Royal Society of Chemistry (RSC) 